\title{
Bifidobacterium longum subspecies infantis: champion colonizer of the infant gut
}

\author{
Mark A. Underwood ${ }^{1,2}$, J. Bruce German ${ }^{2,3}$, Carlito B. Lebrilla ${ }^{2,4}$ and David A. Mills s, $^{2,3}$
}

Oligosaccharides are abundant in human milk. Production of these highly diverse structures requires significant energy expenditure by the mother and yet these human milk oligosaccharides offer no direct nutritive value to her infant. A primary function of human milk oligosaccharides is to shape the infant's intestinal microbiota with life-long consequences. Bifidobacterium longum subspecies infantis (B. infantis) is unique among gut bacteria in its prodigious capacity to digest and consume any human milk oligosaccharide structure, the result of a large repertoire of bacterial genes encoding an array of glycosidases and oligosaccharide transporters not found in other bacterial species. In vitro, B. infantis grows better than other bacterial strains in the presence of human milk oligosaccharides, displays anti-inflammatory activity in premature intestinal cells, and decreases intestinal permeability. In premature infants, $B$. infantis given in combination with human milk increases B. infantis and decreases Enterobacteriaceae in the feces. Probiotics containing $B$. infantis decrease the risk of necrotizing enterocolitis in premature infants. Colonization with B. infantis is also associated with increased vaccine responses. Probiotic organisms have historically been selected based on ease of production and stability. The advantages of B. infantis, selected through coevolution with human milk glycans, present an opportunity for focused manipulation of the infant intestinal microbiota.

$\mathbf{T}$ he colonization of the fetal gut begins in utero with swallowing of amniotic fluid. At that point, infants begin a lifelong relationship with their gut microbiota. Major shifts in the community of microbes inhabiting the intestinal tract (the gut microbiota) and the genes expressed by these microbes (the gut microbiome) and presumably the health consequences of the phenotype of the gut microbiota occur with rupture of the fetal membranes, birth, initiation of feeding, addition of solid foods, weaning, and interventions such as antibiotics, acidsuppression, and prebiotic or probiotic dietary supplements. The predominance of "bifid" microbes in the stools of healthy infants was described more than $100 \mathrm{y}$ ago, prompting the hypothesis that human milk contained "bifidogenic factors" that stimulated the growth of these bifidobacteria (1).

Prebiotics are dietary supplements that promote health benefits by stimulating the growth and/or activity in the gut lumen of commensal microbes (ideally without stimulating potential pathogens); they do not contain live organisms. Probiotics are dietary supplements that do contain live organisms and are intended to promote health benefits through a variety of mechanisms. This article will focus on the coevolution of a collection of complex prebiotic oligosaccharides found in abundance in human milk and a single bacterial subspecies, Bifidobacterium longum subsp. infantis (B. infantis) unique in its capacity to consume these oligosaccharides. Note that the species $B$. longum has two subspecies: $B$. longum subsp. infantis and $B$. longum subsp. longum. These subspecies will be abbreviated in this review as B. infantis and B. longum, respectively.

\section{HUMAN MILK OLIGOSACCHARIDES SHAPE THE INFANT INTESTINAL MICROBIOTA}

Humans stand at the end of the long evolution of mammals, with differences in milk conspicuous for the volume, number of structures, and complexity of milk oligosaccharides (Figure 1) (2-4). Human milk oligosaccharides (HMOs) are the third largest solid component of human milk (after lactose and fat) even in times of famine (5), and yet these free glycans are not digestible by the infant as the human gut does not produce the glycosidases necessary to cleave the HMO linkages. The obvious evolutionary question is: What benefit is provided to the infant that justifies the mother's tremendous expenditure of energy to produce these varied and complex molecules with no apparent nutritional value? The answer to this question comes from careful analyses of the rare capacity of select gut microbes to deconstruct and consume HMOs $(6,7)$. Among multiple microbial species studied, only two genera, Bifidobacterium and Bacteroides, are able to comprehensively utilize HMOs as a primary food source (Table 1) $(8,9)$. This relative resistance to microbial consumption allows the HMOs to arrive intact in the distal small bowel and the colon where the largest numbers of commensal bacteria thrive. 
a
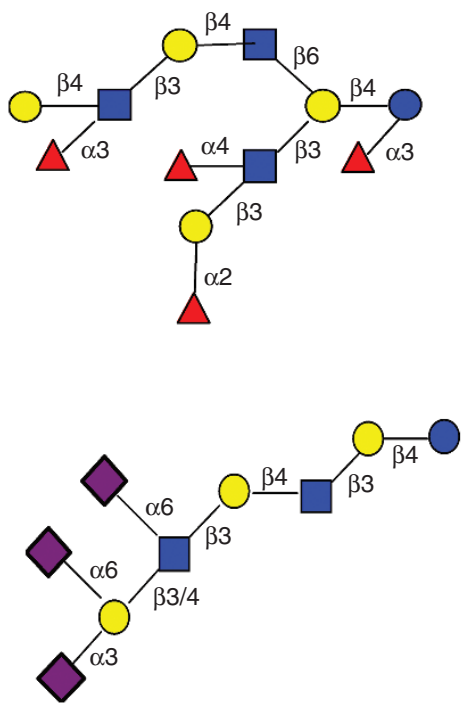

b
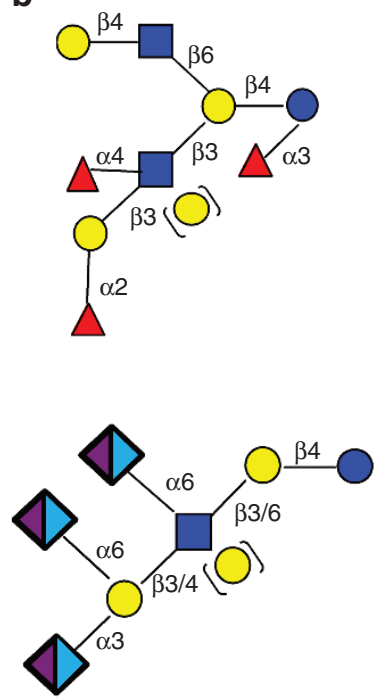

C
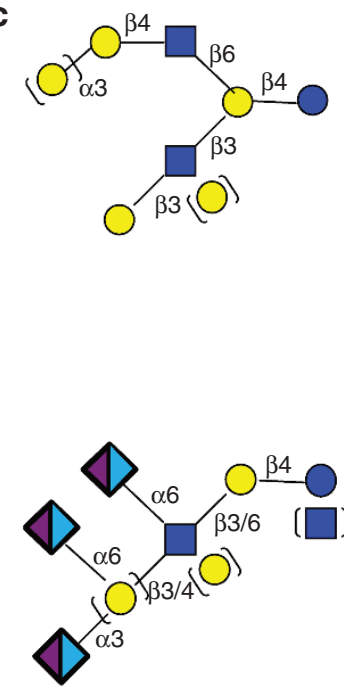

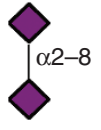

Figure 1. Systematic structural analysis of milk oligosaccharides from multiple mammalian species. Human milk (a) has a higher degree of oligosaccharide polymerization with about $70 \%$ fucosylated structures (upper structure) and less than $20 \%$ sialylated structures (lower structure, exclusively $\mathrm{N}$-acetylneuraminic acid). Nonhuman primate milk (b) varies with species with $20-65 \%$ fucosylated structures (upper structure) and $10-45 \%$ sialylated structures (lower structure, both $\mathrm{N}$-acetylneuraminic acid and $\mathrm{N}$-glycolylneuraminic acid). Other mammals (c) show the least degree of polymerization, less than $5 \%$ fucosylated structures and up to $70 \%$ sialylated structures (lower structure, both $\mathrm{N}$-acetylneuraminic acid and $\mathrm{N}$-glycolylneuraminic acid) $(2,79-83)$. Red triangle $=$ fucose, yellow circle = galactose, blue square $=N$-acetylglucosamine, blue circle $=$ glucose, purple diamond $=\mathrm{N}$-acetylneuraminic acid, and light blue diamond $=\mathrm{N}$-glycolylneuraminic acid .

Table 1. Consumption of single human milk oligosaccharide $(\mathrm{HMO})$ structures by different bacterial species

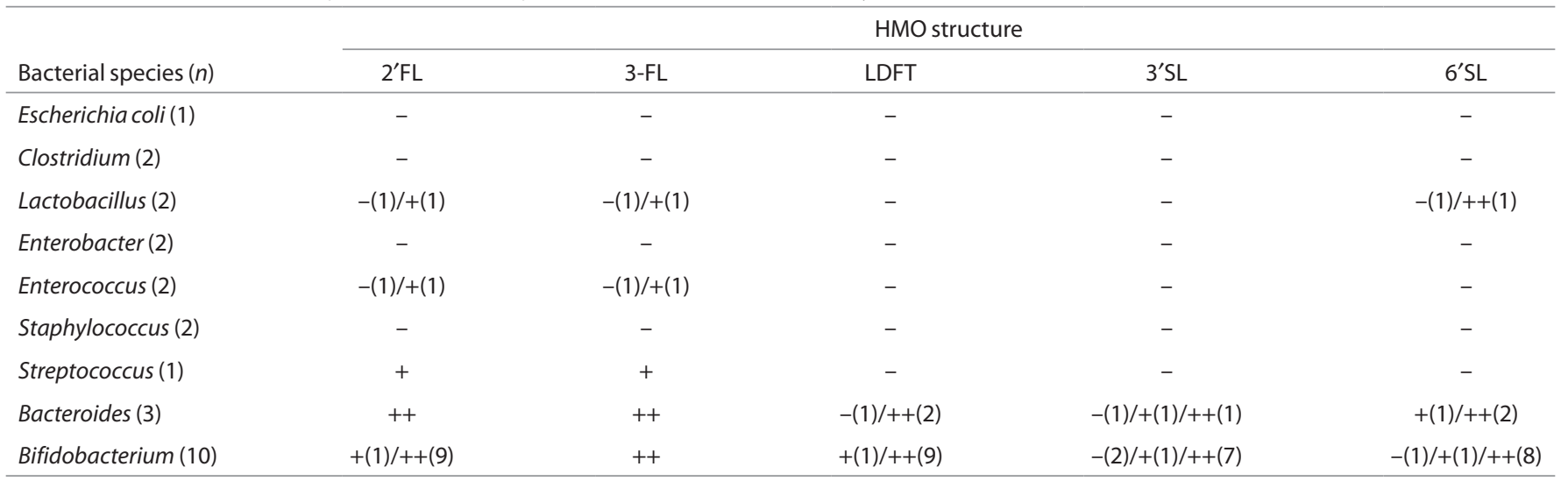

2'FL, 3-FL, and LDFT are abundant fucosylated HMO structures, and 3'SL and 6'SL are abundant sialylated HMO structures. The numbers in parentheses are the number of strains tested. The symbols represent consumption of $<10 \%(-)$, consumption between 10 and $40 \%(+)$, and consumption of $>40 \%$ (++) (from ref. 8 ).

The gut of healthy term infants is initially colonized by bacteria acquired at birth. These "pioneer" bacteria are predominately facultative anaerobes with composition heavily influenced by mode of delivery (10). Within the first days to weeks, two obligate anaerobes, Bacteroides and Bifidobacterium, generally become the most abundant genera (11-13). Previous dogma was that the pioneer bacteria create a low-oxygen environment in which the obligate anaerobes then become dominant; however, two recent observations suggest that this second wave of colonization is more complex. First, species of both Bacteroides and Bifidobacterium are found in maternal feces, human milk, and infant feces suggesting direct inoculation through breastfeeding and maternal-infant contact (14). Second, species of both Bacteroides and Bifidobacterium are aggressive consumers of HMOs (15). Early reports of a relative absence of Bifidobacterium species in the stools of healthy infants (16) were likely due to limitations in methods, e.g., imprecise PCR primers and lack of bead-beating in bacterial DNA extraction (17). Healthy term breastfed infants are colonized by a small number of subspecies including $B$. infantis, $B$. longum, and $B$. breve and to a lesser extent $B$. bifidum and B. pseudocatenulatum, whereas healthy term formula-fed infants are colonized by a more diverse population, including the above species plus bifidobacterial species seen in adults such as $B$. adolescentis (18-20). In adults, increased diversity in the intestinal microbial population is generally considered beneficial (21); however, this may not be the case in the healthy neonate where a predominance of a 


\section{B. infantis: champion colonizer $\mid$ ReView}

few subspecies of bifidobacteria is associated with improved growth (22).

\section{MECHANISTIC EVIDENCE FOR COLONIZATION BY B. INFANTIS}

Early studies demonstrated that a strain of $B$. infantis was better able to grow in a culture medium wherein HMOs were the only carbon source, than strains of $B$. longum, B. breve, or $B$. adolescentis $(23,24)$. The sequencing of this strain of B. infantis demonstrated a large number of genes involved in catabolism of complex carbohydrates (25). Comparison of the closely related subspecies B. longum and B. infantis demonstrated that the former encodes enzymes for the digestion of plant oligosaccharides, while the latter has evolved the capacity to digest HMOs. Most of the strains of $B$. infantis sequenced to date contain a 43 -kb gene cluster (HMO cluster I) that encodes a variety of oligosaccharide transport proteins and glycosyl hydrolases; this gene cluster is not found in other bifidobacterial species $(26,27)$. The one $B$. infantis strain analyzed to date, which showed weak growth in the presence of HMO, has a partial deletion of this gene complex $(24,26)$. Most HMO structures contain either fucose or sialic acid (Figure 1); among species of Bifidobacterium, only $B$. infantis, B. breve, and B. bifidum produce fucosidases and sialidases, and only $B$. infantis is able to digest all $\mathrm{HMO}$ structures (Table 2) (28).

B. infantis strains, when grown in the presence of HMOs, upregulate expression of two groups of bacterial genes. First, transporter proteins that bind to specific HMO linkages, including a number of solute-binding proteins with an affinity for HMOs, are upregulated in B. infantis grown on HMO but not in $B$. infantis grown on the simpler prebiotic oligosaccharides fructo-oligosaccharide or galacto-oligosaccharide $(29,30)$. This suggests that $B$. infantis is able to transport intact HMOs into its cytoplasm and that this capacity is "turned

Table 2. Bifidobacterium species and the number of glycoside hydrolases encoded in their genomes (from ref. 21)

\begin{tabular}{lccc}
\hline Species/subspecies & $\begin{array}{c}\text { Total glycoside } \\
\text { hydrolases }\end{array}$ & $\alpha$-Sialidase & $\begin{array}{c}\alpha-\mathrm{L}- \\
\text { Fucosidase }\end{array}$ \\
\hline B. adolescentis & 22 & 0 & 0 \\
B. angulatum & 13 & 0 & 0 \\
B. bifidum & 17 & 2 & 2 \\
B. breve & 19 & 1 & 1 \\
B. catenulatum & 21 & 0 & 0 \\
B. dentium & 31 & 0 & 1 \\
B. longum subsp. longum & 26 & 0 & 0 \\
B. longum subsp. infantis & 24 & 2 & 5 \\
B. minimum & 2 & 0 & 0 \\
B.pseudocatenulatum & 25 & 0 & 1 \\
B.pseudolongum & 14 & 0 & 1 \\
B. subtile & 3 & 0 & 0 \\
B.thermacidophilum & 9 & 0 & 0 \\
\hline
\end{tabular}

on" by the HMOs. Second, glycosidases with specificity for every linkage in HMOs are upregulated in $B$. infantis grown on HMO. The 16 glycosyl hydrolases expressed by $B$. infantis include $\alpha$-fucosidases, $\beta$-galactosidases, $\beta$-hexosaminadases, and $\alpha$-sialidases, facilitating complete digestion of HMOs within the bacterial cytoplasm that is not possible for other bifidobacteria (6,31-34). B. infantis also differs markedly from B. bifidum and Bacteroides in its specificity for HMOs. $B$. infantis is unable to deconstruct the O-glycans in human mucus in spite of structural similarity to HMOs (30), while B. bifidum and Bacteroides are able to consume both HMOs and mucus glycans (35). Indeed, unlike B. infantis, B. bifidum and Bacteroides species deploy extracellular glycosyl hydrolases that deconstruct complex glycans outside of the cell enabling import and consumption of specific glycan components while other components of the digested glycans (e.g., mono and disaccharides) are left outside the cell (15). In a mouse model, this consumption mode has recently been shown to liberate sugars that promote the growth of pathogens that otherwise would be unable to utilize host glycans (36). These results suggest the hypothesis that Bacteroides species and B. bifidum may not be ideal as probiotics for correction of dysbiosis in human milk-fed premature infants as the byproducts of HMO consumption may stimulate growth of intraluminal pathogens.

HMOs are not the only human milk components of "interest" to B. infantis. Acidic glycolipids (gangliosides) are found arrayed on the surface of fat globules in human milk and may play a role in neurodevelopment (37), pathogen binding within the gut lumen, and shaping the intestinal microbiota (38). Among six species of bifidobacteria tested, B. infantis and B. bifidum were best able to consume the two major gangliosides GM3 and GD3 (39). These data demonstrate that human milk gangliosides have a selective prebiotic effect in addition to that of HMOs. Evolutionary selective pressure has equipped $B$. infantis with multiple enzymes for deconstructing milk glycans, and as a result this subspecies is able to outcompete even other bifidobacteria as well as other commensals and pathogens in the gut lumen of the healthy breastfed infant. This advantage extends beyond free glycans and glycolipids to glycoproteins. In ongoing studies, $B$. infantis produces an endo- $\beta-N$-acetylglucosaminidase that is able to cleave the $\mathrm{N}$-glycans associated with human glycoproteins like lactoferrin, IgA, and IgG. Human milk incubated with this bacterial enzyme undergoes significant $N$-deglycosylation and $B$. infantis grown in the presence of lactoferrin upregulates expression of this enzyme (40). These data suggest two possibilities: that human milk glycoproteins serve a prebiotic role and that these $B$. infantis endoglycosidases release biologically active peptides from human glycoproteins.

\section{B. INFANTIS IN PREMATURE INFANTS}

Premature infants have a markedly different intestinal microbiota than term infants. While term babies progress from colonization with maternal microbes obtained at birth to microbes influenced mostly by diet, premature infants are generally 


\section{Review | Underwood et al.}

colonized with Firmicutes (predominantly staphylococci, streptococci, and enterococci) and Proteobacteria (predominantly Gram-negative Enterobacteriaceae) with a marked absence of bifidobacteria for several weeks or months (41). This dysbiosis (defined as an alteration in the fecal microbiota) in premature infants is likely due to a combination of environmental factors inherent in neonatal intensive care, hygiene, antibiotic use, and endogenous factors including genetics and immaturity of the intestinal immune responses. Dysbiosis appears to be a significant risk factor in susceptibility to necrotizing enterocolitis (NEC), a common and devastating disease that predominantly affects premature infants. Careful studies have demonstrated associations between NEC and early dysbiosis (42), antibiotic administration (43), and acid suppression (44), as well as worsening of dysbiosis just prior to the onset of NEC (45).

Attempts to alter the intestinal microbiota of premature infants with prebiotics alone have yielded mixed results. The incidence of NEC in human milk-fed premature infants is significantly lower than in those receiving formula (46). Milk from mothers delivering preterm does not differ dramatically from milk from mothers delivering at term in numbers of total HMOs; however, the variability of fucosylated HMOs was found to be significantly higher in the former than the latter (47). It is unclear whether the paucity of bifidobacteria in premature infants is due to these small differences in HMO composition, to lack of introduction of bifidobacteria, or to extrinsic factors such as antibiotics and environmental factors. Results to date suggest that any single intervention is insufficient to significantly impact the premature infant gut microbiota. In a small dose escalation trial of added galactooligosaccharide or HMOs in formula-fed premature infants, there were not significant differences in the fecal microbiota with either prebiotic intervention (48). A larger study in premature infants that showed significant changes in the fecal microbiota with antibiotics and only minimal changes with administration of a mixture of galacto-oligosaccharides and fructo-oligosaccharides (49), failed to show significant decreases in the incidence of NEC or sepsis (50) or in neurodevelopment (51) between infants that received the prebiotic mixture and those that received the placebo.

Administration of probiotics to premature infants in most of the clinical trials performed to date is associated with a decreased incidence of NEC $(52,53)$. Routine administration of probiotics to all premature infants has been proposed and is common practice in many countries $(54,55)$. The question of which probiotic product to provide to a premature infant is challenging given the lack of direct comparisons between products and the lack of rigorous standardization of live bacteria as a therapeutic intervention. For example, a high number of discrepancies between the stated contents of commercial probiotic products and the measured contents has been reported $(56,57)$. Furthermore, most current commercial probiotics were developed years ago and selection criteria for organisms were based on stability and ease of industrial production rather than specific mechanistic criteria of the organisms selected. It is now possible to establish standards of strain specificity, dosing accuracy, optimum combinations of paired prebiotics and probiotics, and analysis of changes in the gut microbiota composition. Differences among bifidobacteria illustrate this principle. We compared pure formulations of $B$. infantis and B. animalis subsp. lactis (B. lactis, a common Bifidobacterium in yogurts and commercial probiotics) in dose escalation and cross-over trials and found that $B$. infantis was better able to colonize the intestine than $B$. lactis in both formula-fed and human milk-fed premature infants. In the infants receiving B. lactis, even at high doses, the numbers of total bifidobacteria in the stool were low, and the general bifidobacteria that were present were not the administered species. There was no additive effect of human milk and B. lactis-a result that was not surprising given that the $B$. lactis was chosen for this study because, unlike $B$. infantis, it does not grow in culture medium where HMO is the only carbon source. The highest numbers of fecal bifidobacteria were seen in infants receiving a combination of human milk and B. infantis at a dose of $10^{9}$ organisms twice daily (58). Of the more than 20 published randomized controlled trials of probiotics in premature infants, six have included administration of $B$. infantis alone or in combination; five of these trials showed a decreased incidence of NEC in the probiotic group (53,59-63). A meta-analysis of four studies of administration of $B$. lactis to premature infants showed no decrease in the incidence of NEC (64).

\section{MECHANISMS OF OBSERVED PROTECTIVE EFFECTS}

Recent studies have demonstrated four promising mechanisms by which bifidobacteria decrease the risk of NEC in premature infants. First, as described above, $B$. infantis has a competitive advantage in the presence of human milk components; therefore, increased colonization resulting in decreased diversity of the gut microbiota and fewer luminal pathogens is one likely mechanism of protection. In addition to a selective growth advantage, in vitro studies reveal that $B$. infantis cells grown on HMO bind to cultured intestinal cells at a higher rate suggesting that the unique ability to grow on HMOs coincides with an increased ability to bind and colonize the intestinal mucus layer $(65,66)$.

Second, $B$. infantis has been shown to be anti-inflammatory in several in vitro and animal studies. An immature and poorly modulated immune response to bacterial translocation is believed to be a key trigger of NEC (67). In an elegant series of experiments, explants of both immature and mature human neonatal intestinal tissue were exposed to the supernatant from $B$. infantis. The $B$. infantis supernatant suppressed the exuberant production of the proinflammatory cytokines IL- 6 and IL-8 and toll-like receptors TLR2 and TLR4 triggered by lipopolysaccharide and IL1 $\beta$ in the immature tissue explants. In the mature tissue explants, expression of these cytokines and TLRs was less marked and not significantly different with exposure to the $B$. infantis supernatant. Similar observations were seen with enterocytes from premature infants with NEC and with immature human enterocytes. These experiments suggest that B. infantis produce exogenous substances that promote maturation of the immature innate immune response (68). This 


\section{B. infantis: champion colonizer $\quad$ ReView}

supernatant from B. infantis attenuates Cronobacter sakazakiiinduced enteritis in a newborn mouse model (C. sakazakii is a contaminant of powdered infant formulas associated with both sepsis and NEC in premature infants) (69). In a rat model of NEC, administration of $B$. infantis decreased expression of IL6, IL8, TNF $\alpha$, IL23, and iNOS, decreased the expression of antimicrobial peptides, altered expression of intestinal mucusrelated proteins, and decreased the incidence of NEC (17). In vitro, Caco-2 cell expression of anti-inflammatory IL10 was increased when these cells were exposed to $B$. infantis grown in the presence of HMOs, but not when exposed to B. infantis grown in the presence of lactose. This series of studies suggest that HMOs "turn on" the repertoire of genes in B. infantis within the infant that are important in controlling inflammation (65).

Third, B. infantis decreases intestinal permeability. In mice colonized with human fecal microbes, increased numbers of bifidobacteria are associated with decreased bacterial translocation while increased numbers of Bacteroides and Clostridia are associated with increased bacterial translocation (70). In a neonatal mouse NEC model, B. infantis decreased intestinal permeability, increased stabilization of the tight junction proteins claudin 4 and occludin, and decreased the incidence of NEC (71). In vitro, $B$. infantis grown in the presence of HMO increased expression of junctional-associated molecule (JAM-A) in Caco-2 cells and tight junction protein ZO-1 in HT-29 cells compared with $B$. infantis grown in the presence of lactose, once again confirming that growth on HMOs is necessary to "turn on" genes in B. infantis associated with host intestinal permeability (65).

Fourth, many commensal bacteria produce short chain fatty acids (SCFA, particularly butyrate, propionate, and acetate) with direct and indirect effects on the host. Healthy breastfed infants have higher levels of fecal acetate than formula-fed infants, likely due to increased bifidobacteria. Measurement of fecal SCFA has been proposed as a measure of carbohydrate fermentation and therefore a marker of dysbiosis. However, evaluation of the effects of SCFA is challenging, as these volatile products are both produced and consumed in the colon by bacteria and absorbed by the enterocyte to enter the portal circulation. In adults, increased acetate production may be associated with obesity and inflammation, while butyrate and propionate appear to be protective (72). It has been hypothesized that excessive production of butyrate increases the risk of NEC and limited data from animal models support this hypothesis $(73,74)$. The influence of HMOs, commercial prebiotics, and probiotics on SCFA production remains unclear. In premature infants, administration of $B$. breve was associated with a decrease in fecal butyrate (75), administration of $B$. lactis was associated with increased fecal acetate (76), while administration of a combination product containing $B$. infantis did not alter fecal SCFA compared with placebo (59).

\section{B. INFANTIS IS ASSOCIATED WITH IMPROVED GROWTH AND VACCINE RESPONSES IN TERM INFANTS}

A recent cohort study of infants in Dhaka, Bangladesh found that infants there were heavily colonized with bifidobacteria.
The dominant species of bifidobacteria in these infants $(96 \%$ of whom were breastfed) was $B$. infantis. Correlations among this cohort showed that the infants with the most $B$. infantis in their stools had better weight gain, increased thymic index, and better responses to the oral polio, tuberculosis, and tetanus vaccines (22). The observed correlation does not establish causality. It is possible that the healthiest babies have improved growth, better immune responsiveness, and increased fecal bifidobacteria without the latter causing either of the former; however, these observations support the hypothesis that the composition of the infant microbiota is critical to immune development and surveillance. Probiotic organisms have been demonstrated to boost immune response to polio vaccine in adults (77), but pediatric studies have been equivocal to date, perhaps due to the choice of probiotic strain (78).

\section{CONCLUSION}

HMOs are able to transit the stomach and proximal small bowel of infants without being altered or consumed. In the distal gut, HMOs are selectively consumed by $B$. infantis creating a microbiota that is limited in diversity but associated with improved growth and vaccine responsiveness in term infants and decreased NEC in premature infants. HMOs activate a variety of genes in $B$. infantis that allow it to dominate the gut microbiota and benefit the host by accelerating maturation of the immune response, limiting excessive inflammation, improving intestinal permeability, and increasing acetate production. This symbiotic relationship is a compelling example of coevolution of two species to temporarily protect the full term neonate and nourish a healthy gut microbiota prior to weaning. In the premature infant, this colonization is disrupted and the provision of both human milk and probiotic $B$. infantis appears to be both restorative and protective.

\section{ACKNOWLEDGMENTS}

We gratefully acknowledge all of the researchers in the UC Davis Foods for Health Institute for their enthusiasm, imagination, and collective contribution to this subject matter.

\section{STATEMENT OF FINANCIAL SUPPORT}

This work has been supported by University of California Discovery Grant Program, the UC Davis RISE Program, the California Dairy Research Foundation, Dairy Management Inc., the Bill and Melinda Gates Foundation, and National Institutes of Health awards R01HD059127, R01HD065122, R01HD061923, R21AT006180, R01AT007079, and 1U24DK097154. D.A.M. acknowledges support as the Peter J. Shields Endowed Chair in Dairy Food Science.

Disclosure: Three of the authors (J.B.G., C.B.L., D.A.M.) are the cofounders of Evolve Biosystems, a company focused on diet-based manipulation of the gut microbiota.

\section{REFERENCES}

1. Moro E. Morphologische und biologische Untersuchung über die Darmbakterien des Säuglings [Morphological and biological study of the intestinal bacteria of infants]. Jahrb F Kinderh 1905;61:687-734.

2. Tao N, Wu S, Kim J, et al. Evolutionary glycomics: characterization of milk oligosaccharides in primates. J Proteome Res 2011;10:1548-57.

3. Ruhaak LR, Lebrilla CB. Analysis and role of oligosaccharides in milk. BMB Rep 2012;45:442-51.

4. Aldredge DL, Geronimo MR, Hua S, Nwosu CC, Lebrilla CB, Barile D. Annotation and structural elucidation of bovine milk oligosaccharides 
and determination of novel fucosylated structures. Glycobiology 2013;23: 664-76.

5. Totten SM, Zivkovic AM, Wu S, et al. Comprehensive profiles of human milk oligosaccharides yield highly sensitive and specific markers for determining secretor status in lactating mothers. J Proteome Res 2012;11:6124-33.

6. Sela DA, Mills DA. Nursing our microbiota: molecular linkages between bifidobacteria and milk oligosaccharides. Trends Microbiol 2010;18: 298-307.

7. Garrido D, Dallas DC, Mills DA. Consumption of human milk glycoconjugates by infant-associated bifidobacteria: mechanisms and implications. Microbiology 2013;159(Pt 4):649-64.

8. Yu ZT, Chen C, Newburg DS. Utilization of major fucosylated and sialylated human milk oligosaccharides by isolated human gut microbes. Glycobiology 2013;23:1281-92.

9. Marcobal A, Barboza M, Froehlich JW, et al. Consumption of human milk oligosaccharides by gut-related microbes. J Agric Food Chem 2010;58:5334-40.

10. Dominguez-Bello MG, Costello EK, Contreras M, et al. Delivery mode shapes the acquisition and structure of the initial microbiota across multiple body habitats in newborns. Proc Natl Acad Sci USA 2010;107:11971-5.

11. Boesten R, Schuren F, Ben Amor K, Haarman M, Knol J, de Vos WM. Bifidobacterium population analysis in the infant gut by direct mapping of genomic hybridization patterns: potential for monitoring temporal development and effects of dietary regimens. Microb Biotechnol 2011;4:417-27.

12. Harmsen HJ, Wildeboer-Veloo AC, Raangs GC, et al. Analysis of intestinal flora development in breast-fed and formula-fed infants by using molecular identification and detection methods. J Pediatr Gastroenterol Nutr 2000;30:61-7.

13. Jost $\mathrm{T}$, Lacroix $\mathrm{C}$, Braegger $\mathrm{CP}$, Chassard C. New insights in gut microbiota establishment in healthy breast fed neonates. PLoS One 2012;7:e44595.

14. Jost T, Lacroix C, Braegger CP, Rochat F, Chassard C. Vertical motherneonate transfer of maternal gut bacteria via breastfeeding. Environ Microbiol [online], 2013 (doi:10.1111/1462-2920.12238).

15. Marcobal A, Sonnenburg JL. Human milk oligosaccharide consumption by intestinal microbiota. Clin Microbiol Infect 2012;18:Suppl 4:12-5.

16. Palmer C, Bik EM, DiGiulio DB, Relman DA, Brown PO. Development of the human infant intestinal microbiota. PLoS Biol 2007;5:e177.

17. Underwood MA, Arriola J, Gerber CW, et al. Bifidobacterium longum subsp. infantis in experimental necrotizing enterocolitis: alterations in inflammation, innate immune response, and the microbiota. Pediatr Res 2014;76:326-33.

18. Sakata S, Tonooka T, Ishizeki S, et al. Culture-independent analysis of fecal microbiota in infants, with special reference to Bifidobacterium species. FEMS Microbiol Lett 2005;243:417-23.

19. Roger LC, Costabile A, Holland DT, Hoyles L, McCartney AL. Examination of faecal Bifidobacterium populations in breast- and formula-fed infants during the first 18 months of life. Microbiology 2010;156(Pt 11):3329-41.

20. Turroni F, Foroni E, Pizzetti P, et al. Exploring the diversity of the bifidobacterial population in the human intestinal tract. Appl Environ Microbiol 2009;75:1534-45.

21. Belkaid Y, Hand TW. Role of the microbiota in immunity and inflammation. Cell 2014;157:121-41.

22. Huda MN, Lewis ZT, Kalanetra KM, et al. Stool microbiota and vaccine responses of infants. Pediatrics 2014;134:e362-72.

23. Ward RE, Niñonuevo M, Mills DA, Lebrilla CB, German JB. In vitro fermentability of human milk oligosaccharides by several strains of bifidobacteria. Mol Nutr Food Res 2007;51:1398-405.

24. Locascio RG, Niñonuevo MR, Kronewitter SR, et al. A versatile and scalable strategy for glycoprofiling bifidobacterial consumption of human milk oligosaccharides. Microb Biotechnol 2009;2:333-42.

25. Sela DA, Chapman J, Adeuya A, et al. The genome sequence of Bifidobacterium longum subsp. infantis reveals adaptations for milk utilization within the infant microbiome. Proc Natl Acad Sci USA 2008;105:18964-9.

26. LoCascio RG, Desai P, Sela DA, Weimer B, Mills DA. Broad conservation of milk utilization genes in Bifidobacterium longum subsp. infantis as revealed by comparative genomic hybridization. Appl Environ Microbiol 2010;76:7373-81.
27. Zivkovic AM, German JB, Lebrilla CB, Mills DA. Human milk glycobiome and its impact on the infant gastrointestinal microbiota. Proc Natl Acad Sci USA 2011;108:Suppl 1:4653-8.

28. Sela DA, Mills DA. The marriage of nutrigenomics with the microbiome: the case of infant-associated bifidobacteria and milk. Am J Clin Nutr 2014;99:697S-703S.

29. Garrido D, Kim JH, German JB, Raybould HE, Mills DA. Oligosaccharide binding proteins from Bifidobacterium longum subsp. infantis reveal a preference for host glycans. PLoS One 2011;6:e17315.

30. Kim JH, An HJ, Garrido D, German JB, Lebrilla CB, Mills DA. Proteomic analysis of Bifidobacterium longum subsp. infantis reveals the metabolic insight on consumption of prebiotics and host glycans. PLoS One 2013;8:e57535.

31. Sela DA, Li Y, Lerno L, et al. An infant-associated bacterial commensal utilizes breast milk sialyloligosaccharides. J Biol Chem 2011;286:11909-18.

32. Sela DA, Garrido D, Lerno L, et al. Bifidobacterium longum subsp. infantis ATCC $15697 \alpha$-fucosidases are active on fucosylated human milk oligosaccharides. Appl Environ Microbiol 2012;78:795-803.

33. Yoshida E, Sakurama H, Kiyohara M, et al. Bifidobacterium longum subsp. infantis uses two different $\beta$-galactosidases for selectively degrading type- 1 and type-2 human milk oligosaccharides. Glycobiology 2012;22:361-8.

34. Garrido D, Ruiz-Moyano S, Mills DA. Release and utilization of Nacetyl-D-glucosamine from human milk oligosaccharides by Bifidobacterium longum subsp. infantis. Anaerobe 2012;18:430-5.

35. Marcobal A, Barboza M, Sonnenburg ED, et al. Bacteroides in the infant gut consume milk oligosaccharides via mucus-utilization pathways. Cell Host Microbe 2011;10:507-14.

36. Ng KM, Ferreyra JA, Higginbottom SK, et al. Microbiota-liberated host sugars facilitate post-antibiotic expansion of enteric pathogens. Nature 2013;502:96-9.

37. Park EJ, Suh M, Ramanujam K, Steiner K, Begg D, Clandinin MT. Dietinduced changes in membrane gangliosides in rat intestinal mucosa, plasma and brain. J Pediatr Gastroenterol Nutr 2005;40:487-95.

38. Rueda $\mathrm{R}$. The role of dietary gangliosides on immunity and the prevention of infection. Br J Nutr 2007;98:Suppl 1:S68-73.

39. Lee H, Garrido D, Mills DA, Barile D. Hydrolysis of milk gangliosides by infant-gut associated bifidobacteria determined by microfluidic chips and high-resolution mass spectrometry. Electrophoresis 2014;35:1742-50.

40. Garrido D, Nwosu C, Ruiz-Moyano S, et al. Endo- $\beta$-N-acetylglucosaminidases from infant gut-associated bifidobacteria release complex N-glycans from human milk glycoproteins. Mol Cell Proteomics 2012;11: $775-85$.

41. Westerbeek EA, van den Berg A, Lafeber HN, Knol J, Fetter WP, van Elburg RM. The intestinal bacterial colonisation in preterm infants: a review of the literature. Clin Nutr 2006;25:361-8.

42. Morrow AL, Lagomarcino AJ, Schibler KR, et al. Early microbial and metabolomic signatures predict later onset of necrotizing enterocolitis in preterm infants. Microbiome 2013;1:13.

43. Cotten CM, Taylor S, Stoll B, et al. Prolonged duration of initial empirical antibiotic treatment is associated with increased rates of necrotizing enterocolitis and death for extremely low birth weight infants. Pediatrics 2009;123:58-66.

44. Guillet R, Stoll BJ, Cotten CM, et al. Association of H2-blocker therapy and higher incidence of necrotizing enterocolitis in very low birth weight infants. Pediatrics 2006;117:e137-142.

45. Mai V, Young CM, Ukhanova M, et al. Fecal microbiota in premature infants prior to necrotizing enterocolitis. PLoS One 2011;6:e20647.

46. Meinzen-Derr J, Poindexter B, Wrage L, Morrow AL, Stoll B, Donovan EF. Role of human milk in extremely low birth weight infants' risk of necrotizing enterocolitis or death. J Perinatol 2009;29:57-62.

47. De Leoz ML, Gaerlan SC, Strum JS, et al. Lacto-N-tetraose, fucosylation, and secretor status are highly variable in human milk oligosaccharides from women delivering preterm. J Proteome Res 2012;11:4662-72.

48. Underwood MA, Kalanetra KM, Bokulich NA, et al. Prebiotic oligosaccharides in premature infants. J Pediatr Gastroenterol Nutr 2014;58:352-60.

49. Westerbeek EA, Slump RA, Lafeber HN, et al. The effect of enteral supplementation of specific neutral and acidic oligosaccharides on the faecal microbiota and intestinal microenvironment in preterm infants. Eur J Clin Microbiol Infect Dis 2013;32:269-76. 


\section{B. infantis: champion colonizer}

50. Westerbeek EA, van den Berg JP, Lafeber HN, et al. Neutral and acidic oligosaccharides in preterm infants: a randomized, double-blind, placebocontrolled trial. Am J Clin Nutr 2010;91:679-86.

51. LeCouffe NE, Westerbeek EA, van Schie PE, Schaaf VA, Lafeber HN, van Elburg RM. Neurodevelopmental outcome during the first year of life in preterm infants after supplementation of a prebiotic mixture in the neonatal period: a follow-up study. Neuropediatrics 2014;45:22-9.

52. AlFaleh K, Anabrees J. Probiotics for prevention of necrotizing enterocolitis in preterm infants. Cochrane Database Syst Rev 2014;4:CD005496.

53. Jacobs SE, Tobin JM, Opie GF, et al. Probiotic effects on late-onset sepsis in very preterm infants: a randomized controlled trial. Pediatrics 2013;132:1055-62.

54. Janvier A, Malo J, Barrington KJ. Cohort study of probiotics in a North American neonatal intensive care unit. J Pediatr 2014;164:980-5.

55. Ofek Shlomai N, Deshpande G, Rao S, Patole S. Probiotics for preterm neonates: what will it take to change clinical practice? Neonatology 2014;105:64-70.

56. Marcobal A, Underwood MA, Mills DA. Rapid determination of the bacterial composition of commercial probiotic products by terminal restriction fragment length polymorphism analysis. J Pediatr Gastroenterol Nutr 2008;46:608-11.

57. Aureli P, Fiore A, Scalfaro C, Casale M, Franciosa G. National survey outcomes on commercial probiotic food supplements in Italy. Int J Food Microbiol 2010;137:265-73.

58. Underwood MA, Kalanetra KM, Bokulich NA, et al. A comparison of two probiotic strains of bifidobacteria in premature infants. J Pediatr 2013;163:1585-91.e9.

59. Underwood MA, Salzman NH, Bennett SH, et al. A randomized placebocontrolled comparison of 2 prebiotic/probiotic combinations in preterm infants: impact on weight gain, intestinal microbiota, and fecal short-chain fatty acids. J Pediatr Gastroenterol Nutr 2009;48:216-25.

60. Samanta M, Sarkar M, Ghosh P, Ghosh Jk, Sinha Mk, Chatterjee S. Prophylactic probiotics for prevention of necrotizing enterocolitis in very low birth weight newborns. J Trop Pediatr 2009;55:128-31.

61. Lin HC, Su BH, Chen AC, et al. Oral probiotics reduce the incidence and severity of necrotizing enterocolitis in very low birth weight infants. Pediatrics 2005;115:1-4.

62. Fernández-Carrocera LA, Solis-Herrera A, Cabanillas-Ayón M, et al. Double-blind, randomised clinical assay to evaluate the efficacy of probiotics in preterm newborns weighing less than $1500 \mathrm{~g}$ in the prevention of necrotising enterocolitis. Arch Dis Child Fetal Neonatal Ed 2013;98:F5-9.

63. Bin-Nun A, Bromiker R, Wilschanski M, et al. Oral probiotics prevent necrotizing enterocolitis in very low birth weight neonates. J Pediatr 2005;147:192-6.

64. Szajewska H, Guandalini S, Morelli L, Van Goudoever JB, Walker A. Effect of Bifidobacterium animalis subsp lactis supplementation in preterm infants: a systematic review of randomized controlled trials. J Pediatr Gastroenterol Nutr 2010;51:203-9.

65. Chichlowski M, De Lartigue G, German JB, Raybould HE, Mills DA. Bifidobacteria isolated from infants and cultured on human milk oligosaccharides affect intestinal epithelial function. J Pediatr Gastroenterol Nutr 2012;55:321-7.
66. Kavanaugh DW, O'Callaghan J, Butto LF, et al. Exposure of subsp. to milk oligosaccharides increases adhesion to epithelial cells and induces a substantial transcriptional response. PLoS One 2013;8:e67224.

67. Neu J, Walker WA. Necrotizing enterocolitis. N Engl J Med 2011;364: $255-64$.

68. Ganguli K, Meng D, Rautava S, Lu L, Walker WA, Nanthakumar N. Probiotics prevent necrotizing enterocolitis by modulating enterocyte genes that regulate innate immune-mediated inflammation. Am J Physiol Gastrointest Liver Physiol 2013;304:G132-41.

69. Weng M, Ganguli K, Zhu W, Shi HN, Walker WA. Conditioned medium from Bifidobacteria infantis protects against Cronobacter sakazakiiinduced intestinal inflammation in newborn mice. Am J Physiol Gastrointest Liver Physiol 2014;306:G779-87.

70. Romond MB, Colavizza M, Mullié C, et al. Does the intestinal bifidobacterial colonisation affect bacterial translocation? Anaerobe 2008;14:43-8.

71. Bergmann KR, Liu SX, Tian R, et al. Bifidobacteria stabilize claudins at tight junctions and prevent intestinal barrier dysfunction in mouse necrotizing enterocolitis. Am J Pathol 2013;182:1595-606.

72. Puertollano E, Kolida S, Yaqoob P. Biological significance of short-chain fatty acid metabolism by the intestinal microbiome. Curr Opin Clin Nutr Metab Care 2014;17:139-44.

73. Waligora-Dupriet AJ, Dugay A, Auzeil N, et al. Short-chain fatty acids and polyamines in the pathogenesis of necrotizing enterocolitis: kinetics aspects in gnotobiotic quails. Anaerobe 2009;15:138-44.

74. Nafday SM, Chen W, Peng L, Babyatsky MW, Holzman IR, Lin J. Shortchain fatty acids induce colonic mucosal injury in rats with various postnatal ages. Pediatr Res 2005;57:201-4.

75. Wang C, Shoji H, Sato H, et al. Effects of oral administration of Bifidobacterium breve on fecal lactic acid and short-chain fatty acids in low birth weight infants. J Pediatr Gastroenterol Nutr 2007;44:252-7.

76. Mohan R, Koebnick C, Schildt J, Mueller M, Radke M, Blaut M. Effects of Bifidobacterium lactis Bb12 supplementation on body weight, fecal $\mathrm{pH}$, acetate, lactate, calprotectin, and IgA in preterm infants. Pediatr Res 2008;64:418-22.

77. de Vrese M, Rautenberg P, Laue C, Koopmans M, Herremans T, Schrezenmeir J. Probiotic bacteria stimulate virus-specific neutralizing antibodies following a booster polio vaccination. Eur J Nutr 2005;44:406-13.

78. Maidens C, Childs C, Przemska A, Dayel IB, Yaqoob P. Modulation of vaccine response by concomitant probiotic administration. Br J Clin Pharmacol 2013;75:663-70.

79. Wu S, Tao N, German JB, Grimm R, Lebrilla CB. Development of an annotated library of neutral human milk oligosaccharides. J Proteome Res 2010;9:4138-51.

80. Wu S, Grimm R, German JB, Lebrilla CB. Annotation and structural analysis of sialylated human milk oligosaccharides. J Proteome Res 2011;10: 856-68.

81. Urashima T, Saito T, Nakamura T, Messer M. Oligosaccharides of milk and colostrum in non-human mammals. Glycoconj J 2001;18:357-71.

82. Boehm G, Stahl B. Oligosaccharides. In: Mattila-Sandholm T, Saarela M, eds. Functional Dairy Products. Cambridge, England: CRC Press, 2003:203-243.

83. Tao N, DePeters EJ, Freeman S, German JB, Grimm R, Lebrilla CB. Bovine milk glycome. J Dairy Sci 2008;91:3768-78. 